\title{
AMENDMENTS
}

\section{Author Correction: Perceptual straightening of natural videos}

Olivier J. Hénaff ${ }^{0}$, Robbe L. T. Goris and Eero P. Simoncelli

Correction to: Nature Neuroscience https://doi.org/10.1038/s41593-019-0377-4, published online 29 April 2019.

In the version of this article initially published online, labels in Fig. 6b,d were switched. Green dots should have been labeled "Artificial" and orange dots should have been labeled "Contrast". The error has been corrected in the print, PDF and HTML versions of this article.

Original
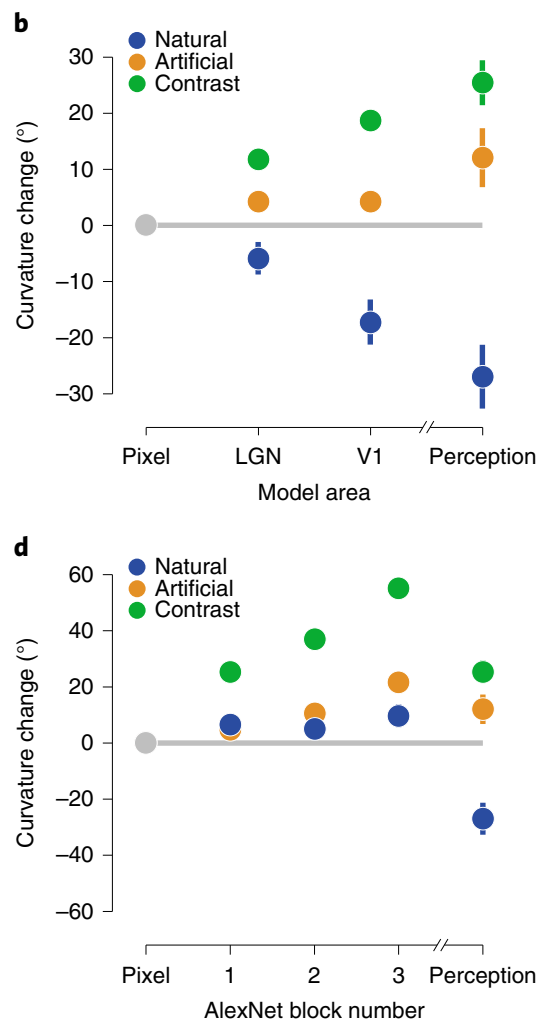

Corrected
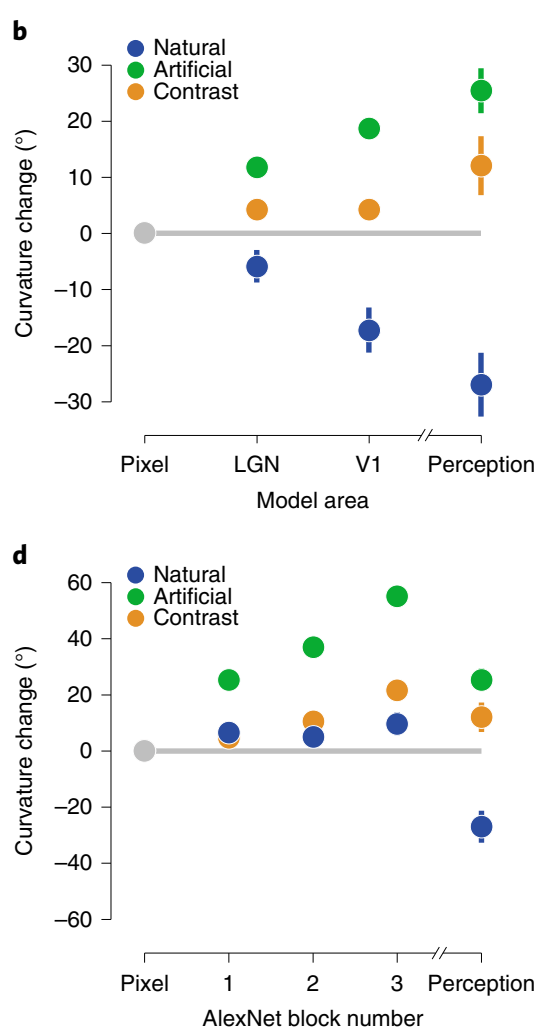

Fig. 6b,d | Original and Corrected.

Published online: 15 May 2019

https://doi.org/10.1038/s41593-019-0420-5

\section{Publisher Correction: Human cognition involves the dynamic integration of neural activity and neuromodulatory systems}

James M. Shine (D), Michael Breakspear (1), Peter T. Bell, Kaylena A. Ehgoetz Martens, Richard Shine, Oluwasanmi Koyejo, Olaf Sporns (iD) and Russell A. Poldrack (1)

Correction to: Nature Neuroscience https://doi.org/10.1038/s41593-018-0312-0, published online 21 January 2019.

In the version of this article initially published, Kaylena A. Ehgoetz Martens' name was misspelled as Kayla. The error has been corrected in the HTML and PDF versions of the article. 